\title{
La prévision de la demande, un outil stratégique négligé par les détaillants
}

\author{
Joëlle Bouchard $^{\mathrm{a}}$
}

RÉSUMÉ. Cet article traite de l'importance et des avantages de l'utilisation des prévisions comme outil d'aide à la décision dans l'estimation de la demande future pour l'industrie du commerce de détail. Des sondages, réalisés à plusieurs reprises dans le temps, montrent que son utilisation demeure plutôt faible dans les organisations malgré le développement récent de nouveaux outils dont l'efficacité et la précision sont démontrées. Les raisons de cette sous-utilisation et des pistes de solutions sont présentées dans cet article.

\begin{abstract}
This paper discusses the importance and benefits of using forecasts as a decision support tool in estimating future demand for the retail industry. Surveys, conducted over time, show that its use remains rather weak in organizations despite the recent development of new tools whose efficiency and accuracy are demonstrated. The reasons for this underutilization and possible solutions are presented in this paper.
\end{abstract}

\section{Introduction}

Les mauvaises nouvelles s'accumulent, le commerce de détail est mal en point. De nombreuses bannières ont récemment fermé leurs portes. Après Jacob, Mexx, Cuir Danier, Smart Set, Target au Canada, Future Shop et La Baie d'Hudson, c'est maintenant au tour de Sears de mettre fin à ses activités après plus de 64 ans d'opération. À l'évidence, l'industrie du commerce de détail est en plein bouleversement.

Les détaillants, pour assurer leur pérennité, doivent constamment s'adapter aux goûts et besoins changeants des clients, aux nouvelles tendances du moment, à l'évolution des comportements d'achat des consommateurs notamment avec l'achat en ligne, à la forte concurrence et à la situation économique. Le statu quo n'est plus une option, l'adaptation aux changements est la clé de voûte pour survivre et prospérer.

Or, cette adaptation aux changements oblige les entreprises à mieux comprendre et estimer la demande future afin de proposer aux clients les produits qui répondent à leurs attentes, à un prix

a Professeure, Ph. D., Université du Québec à Chicoutimi raisonnable, tout en s'assurant de disposer d'un inventaire adéquat pour éviter les pénuries et le surstockage. La priorité des entreprises est donc la satisfaction de la demande des clients et constitue leur principal cheval de bataille pour survivre dans un environnement fortement concurrentiel.

L'estimation et la prévision de la demande sont des exercices extrêmement difficiles dans un contexte où les marchés sont particulièrement volatils, où la concurrence est omniprésente, où les cycles de vie des produits de consommation sont de plus en plus courts et où la demande est influencée par une combinaison d'effets saisonniers, de jours spéciaux (le vendredi fou, Noël, la Saint-Valentin, etc.), de promotions et même de conditions météo.

Les défis sont de taille pour les entreprises du commerce de détail. Celles en mesure de bien saisir les premiers signaux du marché seront mieux outillées pour prévoir la demande et prendre des décisions plus éclairées en réponse aux besoins changeants des consommateurs. 


\section{La prévision de la demande : un outil stratégique}

La prévision de la demande consiste à estimer la consommation future de produits offerts par les détaillants. Les avantages d'une bonne prévision de demande au sein des organisations sont nombreux. Une meilleure connaissance des nouvelles occasions de marché, une augmentation de la rapidité à répondre à la demande, une amélioration de la satisfaction de la clientèle, la réduction des inventaires, une diminution de l'obsolescence des produits, une optimisation des opérations de distribution, un ordonnancement de la production plus efficace, une planification des ressources humaines facilitée et une meilleure anticipation des besoins en capital et financements futurs ne sont que quelques exemples (Marien, 1999; McIntyre et al., 1993).

Nul ne doute alors de l'importance d'élaborer des prévisions de demande fiables. À cet effet, la littérature scientifique présente une très grande variété de méthodes de prévision adaptées aux diverses problématiques, aux types et particularités des données utilisées (demande intermittente, cycle de vie court, données limitées, produits ayant une fin de vie planifiée, plusieurs saisonnalités affectant la demande, etc.) dans l'élaboration des prévisions et aux différences contextuelles propres aux entreprises. Les lecteurs intéressés par un état de l'art des différentes méthodes de prévision développées au fil des ans sont invités à lire Armstrong (1984), Fildes (1985), Winklhofer et al. (1996), Gardner (2006) ainsi que De Gooijer et Hyndman (2006).

Les 10 dernières années ont été particulièrement florissantes en matière de développement de nouveaux outils de prévision. Grâce au progrès de la technologie informatique, les grandes entreprises emmagasinent, chaque jour, des millions d'informations provenant des différents points de vente dans un réseau de détaillants. Ces informations portent sur les transactions de vente, les inventaires de produits, le nombre de magasins, les promotions, les commandes, le prix, le nombre de clics sur une page Web, de même que des données relatives aux conditions météo. Nous sommes à l'ère du big data et de l'intelligence artificielle. Les chercheurs tirent profit de cette immense quantité d'informations et développent des outils modernes d'exploration de données (data mining), d'apprentis- sage automatique (machine learning) et d'analyse prédictive qu'ils combinent aux méthodes d'estimation de demande future afin d'améliorer significativement la qualité des prévisions. Les outils d'aide à la décision disponibles sont de plus en plus sophistiqués et accessibles. Les résultats probants documentés dans la littérature permettent d'imaginer l'avenir du commerce de détail sous des jours meilleurs.

\section{La prévision de la demande : un outil négligé}

Les avantages démontrés des prévisions, depuis plusieurs décennies, laissent supposer que toutes les entreprises sont convaincues de leur utilité et de la nécessité d'adopter ces méthodes de prévision comme outils d'aide à la décision dans les activités quotidiennes des organisations. Or, des études indiquent qu'en pratique, peu d'entreprises mettent en application ces outils dont l'efficacité et la précision ne font pourtant aucun doute.

À cet effet, Dalrymple (1987) a procédé à un important sondage aux États-Unis dans le but de connaitre les pratiques en matière de prévision de vente au sein d'entreprises américaines des secteurs industriel et commercial. Le faible taux de participation des entreprises commerciales de 5,41\% laisse supposer qu'il y a 30 ans, leur utilisation était plutôt négligeable. Les résultats de cette enquête démontrent que, pour les entreprises commerciales, les prévisions subjectives et la méthode d'extrapolation naïve étaient les plus largement utilisées dans des proportions respectives de $19,6 \%$ et $17,4 \%$. À titre informatif, la prévision subjective est une méthode qualitative basée sur l'opinion d'experts dans le but de connaitre l'évolution probable de la demande future. Une prévision naïve est une méthode quantitative qui consiste à estimer la demande de la période future à partir de la demande réelle de la période précédente. À titre illustratif, la demande prévue demain est estimée à partir de la demande connue d'aujourd'hui ou encore la demande de la semaine prochaine est estimée à partir des informations de celle de cette semaine.

Un nouveau sondage, mené par Sanders et Manrodt (1994), avait pour objectif d'identifier les raisons poussant les gestionnaires de firmes américaines à utiliser aussi massivement les méthodes de 
prévision subjectives alors que les méthodes quantitatives sont largement enseignées dans les écoles commerciales et leur précision démontrée. Par ailleurs, la disponibilité des ordinateurs et des logiciels de prévision, de plus en plus accessibles, explique difficilement le faible taux d'utilisation des méthodes quantitatives dans l'élaboration des prévisions de vente futures. Avec un faible taux de réponse de $19,2 \%$ à ce sondage, les auteurs ont tout de même dégagé le constat suivant : les gestionnaires sont de plus en plus familiers avec les méthodes de prévision quantitatives, mais, en dépit de cela, leur utilisation n'est pas plus répandue. Les principaux obstacles formulés sont d'une part, le manque de données quantitatives pertinentes rendant ainsi l'application d'outils de prévision difficile et, d'autre part, le faible soutien de la part des organisations dans la mise en application de ces méthodes. Par ailleurs, on note que les grandes entreprises qui possèdent un chiffre d'affaires élevé ont plus souvent recours à des méthodes quantitatives plus sophistiquées qu'à des méthodes naïves. Les budgets plus importants, de meilleurs outils informatiques et un plus grand nombre de produits à prévoir expliquent, en partie, ce phénomène.

McCarthy et al. (2006) ont proposé une synthèse des différents sondages réalisés entre 1984 et 2001 sur l'utilisation des outils de prévision en entreprise. Le constat demeure le même, plusieurs années plus tard. En effet, on remarque un développement technologique qui facilite l'acquisition d'un grand nombre d'informations sur la demande et sur les facteurs exogènes qui l'influencent, un meilleur accès aux logiciels commerciaux spécialisés en prévision et de nouvelles méthodes de prévision toujours plus sophistiquées mises à la disposition des prévisionnistes au sein des organisations. Mais étonnamment, l'utilisation des techniques de prévision ne s'est pas généralisée pour autant.

Plus récemment, Industrie Canada a publié le rapport canadien 2010 sur l'état du commerce de détail. On y apprend que seuls $42 \%$ des entreprises canadiennes parmi les plus performantes de l'industrie utilisent des modèles de prévision pour estimer la demande future. Ces entreprises génèrent, pour la plupart, des prévisions au moyen de logiciels de prévision et de planification de la demande. L'utilisation de logiciels commerciaux de prévision tels Forecast Pro, SmartForecaster, Forecast management, etc. est fréquente en raison de la simplicité de leur utilisation. Thomassey $(2002,2010,2014)$ dresse, à cet effet, une liste des différents logiciels commerciaux les plus utilisés par les entreprises œuvrant dans l'industrie du commerce de détail.

Encore aujourd'hui, les arguments justifiant l'utilisation de prévisions subjectives pour prévoir la demande future sont tenaces. Gerussi (2017) identifie cinq idées préconçues sur les prévisions de vente qui perdurent et qui permettraient d'expliquer les réticences des gestionnaires d'entreprises à utiliser des outils de prévisions quantitatives.

1- Il est inutile de faire des prévisions de vente puisque celles-ci sont admises comme étant toujours fausses.

2- On ne peut pas prévoir quand les ventes sont très faibles.

3- Il y a trop de facteurs à prendre en compte pour pouvoir faire de bonnes prévisions de vente.

4- On a besoin d'une seule prévision de vente pour tous les usages dans l'entreprise;

5- Utiliser des prévisions de vente, c'est compliqué.

Un des objectifs de l'élaboration des prévisions est de permettre une meilleure compréhension du comportement de la demande pour des produits de consommation, d'établir des liens de dépendance entre certaines variables (par exemple le volume des ventes d'une journée et le jour de la semaine ou encore le nombre d'unités vendues et le prix demandé) et d'élaborer des probabilités de réalisation de demande future qui alimenteront le processus de planification et la prise des décisions d'affaires (ajuster l'offre de produits, déterminer la quantité d'unités à garder en inventaire, choisir les bons canaux de distribution, cibler la meilleure stratégie marketing, etc.).

Or, il est vrai que les prévisions sont toujours fausses et difficiles à établir, mais les avantages qu'elles procurent en termes d'amélioration de la performance des organisations justifient son utilisation au détriment d'approches intuitives d'estimation des ventes futures.

De toute évidence, il existe un décalage évident entre la théorie et la pratique dans le domaine des prévisions de demande. La partie qui suit tente de mieux comprendre les raisons et d'identifier des pistes de solution. 


\section{Pistes de solution}

Certains facteurs permettent d'expliquer l'écart entre la théorie et la pratique. Notons d'abord, selon Melzi (2016), que trop souvent les entrepreneurs ne sont pas suffisamment formés et sensibilisés à l'importance des outils de prévision et des avantages concurrentiels potentiels. De plus, bon nombre d'entre eux ne sont pas de fervents utilisateurs d'outils statistiques et ne disposent donc pas d'informations quantitatives sur la situation du marché, qui pourraient pourtant leur être d'une grande utilité. Par exemple, la participation à des regroupements d'entrepreneurs ou à des chambres de commerce pourrait les aider à acquérir une meilleure formation. Le lancement d'une campagne de sensibilisation vouée à la promotion des entreprises ayant implanté avec succès des outils prévisionnels afin de créer un système d'émulation est aussi une avenue intéressante à explorer.

Les entrepreneurs savent que des logiciels de prévision existent, mais leur utilisation nécessite une connaissance technique des outils de prévision et de leur paramétrage et malheureusement, pour plusieurs, cela ne fait pas partie de leur champ de compétences. Aussi, un soutien technique mieux adapté aux besoins des organisations dans le but de faciliter leur autonomie s'avère indispensable.

L'élaboration de programmes gouvernementaux pour soutenir les organisations dans l'implantation d'outils de prévision de la demande et l'acquisition de logiciels adaptés à l'industrie du commerce de détail constituent des propositions d'action avantgardistes et souhaitables pour faciliter la prise de décision d'affaires.

L'organisation de séminaires ou de formations collégiales ou universitaires, adaptés aux besoins des entrepreneurs actuels et futurs visant l'acquisition des connaissances en analyse de données, en analyse prédictive et en intelligence d'affaires doit être envisagée. L'objectif étant de leur permettre d'instaurer, au sein de leur entreprise, une culture numérique indispensable dans l'environnement actuel d'affaires.

Par ailleurs, trop peu d'organisations acceptent de divulguer des données réelles d'entreprises au grand dam des chercheurs. À défaut, les nouvelles méthodes de prévision sont alors testées sur des séries chronologiques simulées ou provenant de banques utilisées lors de compétitions entre prévisionnistes. Ce partage d'informations pertinentes entre les entreprises de divers secteurs d'activité et les chercheurs aurait l'avantage de créer un lien entre les entités et de mieux définir les conditions d'application, les avantages et les limites des méthodes de prévision facilitant ainsi leur implantation au sein des organisations.

\section{Conclusion}

À la lumière de cette analyse, il est évident que les gestionnaires ont besoin de méthodes de prévision faciles à utiliser et à comprendre, dont la précision est démontrée, le tout, intégré dans un système informatisé d'aide à la décision.

Il est donc sous la responsabilité des gestionnaires, des chercheurs et des développeurs de logiciels de créer des liens et de travailler étroitement ensemble afin de développer des outils de prévision fiables, faciles à comprendre et à implanter.

Il est par ailleurs de la responsabilité des gouvernements d'aider les entreprises à prospérer en leur permettant d'adopter les outils modernes de gestion et de s'ajuster aux nouvelles réalités du marché.

\section{RÉFÉRENCES}

Armstrong, J. S. (1984). Forecasting by extrapolation: Conclusions from 25 years of research. Interfaces, 14(6), 52-66.

Bouchard, J. (2016). Prévision et réapprovisionnement dynamiques de produits de consommation à cycle rapide. (These de doctorat inédite). Université Laval.

Fildes, R. (1985). Quantitative forecasting - The state of the art: Econometric models. Journal of the Operational Research Society, 36, 549-580.

Dalrymple, D. J. (1987). Sales forecasting practices: results from an United States survey. International Journal of Forecasting, 3 , 379-391.

De Gooijer, J. G., Hyndman, R. J. (2006). 25 years of time series forecasting. International Journal of Forecasting, 22, 443-473. 
Gardner, E. S. (2006). Exponential smoothing: The state of the art - Part II. International Journal of Forecasting, 22, 637-666.

Gerussi, A. (2017, mars). 5 idées reçues sur la prévision des ventes. Supply Chain Magażine.

Marien, E. J. (1999). Demand planning and sales forecasting: a supply chain essential. Supply Chain Management Review, 1-17.

Melzi, Z. (2016). Un outil indispensable, mais négligé. El Watan. Repéré à www.djazairess.com/fr/elwatan/511468.

McCarthy, T. M., Davis, D. F., Golicic, S. L., Mentzer, J. T. (2006). The evolution of sales forecasting management : a 20-year longitudinal study of forecasting practices. Journal of Forecasting, 25, 303-324.

McIntyre, S. H., Archabal, D. D., Miller, C. M. (1993). Applying case-based reasoning to forecasting retail sales. Journal of Retailing, 69, 372-398.

Sanders, N. R., Manrodt, K. B. (1994). Forecasting practices in U.S. corporations: Survey results. Interfaces, 24, 92-100.

Thomassey, S. (2002). Méthodologie de la prévision des ventes appliquée à la distribution textile. (Thèse de doctorat inédite). Université des sciences et technologies de Lille.

Thomassey, S. (2010). Sales forecasts in clothing industry: The key success factor of the supply chain management. International Journal of Production Economics, 128, 470-483.

Thomassey, S. (2014). Sales forecasting in apparel and fashion industry : a review. Intelligent fashion forecasting systems: models and applications. Heidelberg, Allemagne: Springer-Verlag Berlin.

Winklhofer, H., Diamantopoulos, A., Witt, S. F. (1996). Forecasting practice: a review of the empirical literature and an agenda for future research. International Journal of Forecasting, 12, 193-221. 